\title{
Quasiclassical Random Matrix Theory
}

\author{
R. E. Pranged \\ Institute de Physique Nucléaired, Orsay, France \\ Laboratoire de Physique Quantiqued, Université Paul Sabatier, Toulouse, France
}

(May 12, 1996)

We directly combine ideas of the quasiclassical approximation with random matrix theory and apply them to the study of the spectrum, in particular to the two-level correlator. Bogomolny's transfer operator $T$, quasiclassically an $N \times N$ unitary matrix, is considered to be a random matrix. Rather than rejecting all knowledge of the system, except for its symmetry, [as with Dyson's circular unitary ensemble], we choose an ensemble which incorporates the knowledge of the shortest periodic orbits, the prime quasiclassical information bearing on the spectrum. The results largely agree with expectations but contain novel features differing from other recent theories.

The study of wave systems of low symmetry is known, somewhat misleadingly, as "quantum chaos". Aside from experiment and numerics, the most important tools in this field are the quasiclassical approximation [QCA] [1], and random matrix theory [RMT] [2].

These two approaches are complementary. Standard RMT treated problems where knowledge of the Hamiltonian is almost nil. It identifies universal features, notably of the spectral statistics, which statistically persist for all quantum systems with time reversal invariance, say.

Standard RMT fails for the most fundamental spectral statistic, however, namely the mean or smoothed [Weyl] density of states, $\bar{d}(E)$, which is not, after all, so broadly universal. However, it is argued that local quantities can be calculated, i.e. quantities involving energies in the range $E \pm W$, where $W \bar{d}(E)>>1$, and $W$ is small enough so that $\bar{d}(E)$ can be taken as constant. RMT treats the Hamiltonian, $\mathcal{H}$, as a random $N \times N$ matrix, locally normalizes to $\bar{d}$, and lets $N \rightarrow \infty$.

In the QCA, a system is chosen, e.g. a particular stadium billiard, and the smallness of the ratio [wavelength $] /[$ characteristic classical length] is exploited. A particular spectrum, not just statistics, is in principle obtained. The basic idea, of Gutzwiller [3] but much advanced by others [4], [5], [6], [7], makes two subtly related but ostensibly different QCA approximations.

The first is the Weyl approximation to $\bar{d}$ whose leading order is the phase space integral $\bar{d}(E)=$ $\frac{1}{(2 \pi \hbar)^{f}} \int d^{f} p d^{f} q \delta(E-\mathcal{H}(p, q))$ where the dimension $f$ is here taken to be 2 . The density of states is divided into $d(E) \equiv \sum \delta\left(E-E_{a}\right)=\bar{d}(E)+d_{o s c}(E) . \quad \mathrm{A}$ WKB-like approximation is made to give $d_{o s c}$, describing the oscillations about the Weyl term, $d_{\text {osc }}(E)=$ $\sum_{p} A_{p} \cos S_{p}(E) / \hbar$. The sum is over periodic orbits of ac- tion $S_{p}$ [which includes the Maslov index] and $A_{p}$ depends on the classical stability of the orbit. In this primitive form, the result is the Gutzwiller trace formula [3].

Many advances have been made, e.g. $d_{\text {osc }}$ can be written as a ratio of absolutely convergent series, rather than as the divergent series above [8]. The major result is that by such means only orbits whose period $T_{p}=\partial S_{p} / \partial E$ is less than half the "Heisenberg time", $\tau_{\hbar}=2 \pi \hbar d \propto 1 / \hbar$ need be considered: All longer orbits can be effectively expressed in terms of these. Orbits longer than $\tau_{\hbar}$ combine to give nothing, those shorter display a sort of reflection symmetry [resurgence] about $\frac{1}{2} \tau_{\hbar}$.

No direct way to solve a fundamental problem is known, however. Namely, the number of periodic orbits proliferates exponentially with $T_{p}$ and for small $\hbar$ no one can actually find such long orbits, let alone add them up. Therefore recourse is made to a statistical treatment of the long orbits, via the Hannay-Ozorio de Almeida sum rule [9], valid for hard chaotic systems. This requires a form of the diagonal approximation [DA]. [The product $d_{o s c} d_{o s c}$ is expanded in a double Gutzwiller series, and only the diagonal terms are kept. After the DA, quantum effects enter only trivially through the energy scale $\bar{d}$.] The DA by itself is not sufficient, and it fails dramatically [at long time] since long orbits need to show important correlations, if they are to describe a discrete spectrum, and give results in agreement with RMT and experiment for neighboring level properties [10]. [ A recent effort of Bogomolny and Keating [11] builds in the discreteness of the spectrum in an ad hoc but effective way. The supersymmetry [SS] technique has begun to find good results in this connection also [12.] In short, the QCA has to invoke statistics to deal with the long orbits. On the other hand, there are many influences of the short periodic orbits on the spectrum which are observable and have been of great interest.

A crucial advance in semiclassics was made by Bogomolny [6] with his $T$ operator. The $T$ operator is an approximation to and/or generalization of the boundary integral method used to study billiard problems. It is a QCA to an exact kernel $K\left(q, q^{\prime}, E\right)$ [where $q, q^{\prime}$ run over the boundary of the billiard] such that the spectrum is given by the zeroes of $\operatorname{det}(1-K(E))$. The equivalence of the 'resurgence' mentioned above was shown: Namely, in QCA $T$ is unitary and of rank $N$, i.e. equivalent to an $N \times N$ unitary matrix $T_{m n}(E)$, Further, a general Poincaré surface of section can be used instead of the boundary, extending the technique beyond billiards.

Our proposal is to treat the matrix $T_{m n}$ as a ran- 
dom unitary matrix, generalizing Dyson's famous work [2]. This has several advantages over the usual choice of the Hamiltonian as random matrix.

- The mean distribution of eigenvalues $e^{i \theta_{n}}, \bar{d}(\theta)$, is physically meaningful, and related to $\bar{d}(E)$. [We distinguish the distributions in angle and energy by the argument. We also denote, by $\bar{d}$ without an argument, the Weyl density of states. Dyson's COE and CUE matrix ensembles have $\bar{d}(\theta)=N / 2 \pi$.] The study of deviations from $\bar{d}$ constant is the purpose of $d_{o s c}$. Random Hamiltonian matrices are unable to deal with such questions.

- The rank $N$ is meaningful. It is [usually] given by $N \simeq 2 L / \lambda(E)$ where $L$ is the length of the surface of section, and $\lambda$ is the wavelength at energy $E$.

- The matrix size is $N \propto \hbar^{-1}$ rather than $\hbar^{-2}$, an important efficiency of the boundary integral method.

- Specific quasiclassical knowledge may be incorporated into the definition of the ensemble of matrices in a natural and convenient way. In particular, one may study modified Dysonian ensembles, which have prescribed mean values of the traces $\sigma_{r}=\operatorname{Tr} T^{r}$. The set of traces $\sigma_{r}, r=1 . . N$ uniquely determine $\operatorname{det}(1-T)$ and thus the spectrum. Low order traces are usually well evaluated quasiclassically by the method of stationary phase. They are expressed in terms of short periodic orbits, and we take this approximation for $\sigma_{r}$. The high order traces cannot usually be so evaluated as the number of orbits is too large.

Some difficulties with the approach are

- Direct calculation gives the eigenphases $\theta_{n}$ rather than the energies $E_{a}$ for which $\theta_{n}\left(E_{a}\right)=0 \bmod 2 \pi$. However, there is evidence that the $\theta_{n}$ are locally linear functions of the energy. For $N$ large, this is sufficient, as Dyson showed. [Bogomolny, in unpublished lectures, has made this connection more precise.] For billiards one can probably do better, since scaling indicates that $\theta_{n}=a\left(k-k_{n}\right)$ where $k=\sqrt{E}$. [We put $\hbar=1$.]

- We have assumed that $N$ is an integer which depends on energy. Therefore, rather unphysical jumps in $N$ are needed as the energy increases. Perhaps the simplest way around this is to express the results as a function of $N$ and 'analytically continue' to continuous $N$.

Let $Z(E)=\operatorname{det}(1-T)=\prod\left(1-e^{i \theta_{n}}\right)$. Then

$$
\begin{aligned}
d_{o s c} & =\frac{-1}{\pi} \operatorname{Im} \frac{d}{d E} \ln Z(E)=-\frac{1}{2 \pi} \sum_{n} \theta_{n}^{\prime}\left(1+\operatorname{Im} \cot \frac{1}{2} \theta_{n}\right) \\
& =\sum_{n}\left[\frac{-1}{2 \pi} \theta_{n}^{\prime}+\theta_{n}^{\prime} \delta\left(\theta_{n}(E)\right)\right]
\end{aligned}
$$

Assuming $\theta_{n}(E)=\left(E-E_{n}\right) \theta_{n}^{\prime}$ with $\theta_{n_{-}}^{\prime} \approx \theta^{\prime}$ approximately independent of $n$, gives $\bar{d}=\bar{d}_{\text {Weyl }}(E)=$ $\frac{1}{2 \pi} \sum_{n} \theta_{n}^{\prime}=\frac{N}{2 \pi} \theta^{\prime}$. For a billiard, $\theta^{\prime}=a / 2 k$. Using the estimate for $N$ it is found that $\bar{d}=L a / 4 \pi^{2}$. Thus $a=A \pi / L$, where $A$ is the area of the billiard.

We now add knowledge of the short period orbits and only this knowledge to the problem, in the spirit of the information theoretical approach of Balian [13]. We cite other recent work using this technique [14]. Let $d \mu(T)$ be the measure for the class of random matrices under consideration. We want the probability distribution $P(T) d \mu(T)$. Then the 'entropy' $S[P]=$ - $\int d \mu(T) P(T) \ln (P(T))$ is maximized, subject to conditions $\int P(T) \operatorname{Tr} T^{r} d \mu(T)=\sigma_{r} ; r=1,2,$. .

For simplicity we choose the measure without time reversal symmetry corresponding to Dyson's CUE. $T$ can be parametrized by its $N$ eigenphases $\theta_{n}$ together with $N(N-1)$ other real parameters $b_{s}$, which we integrate out. Then $d \mu(T)=\prod_{n<m}\left|e^{i \theta_{m}}-e^{i \theta_{n}}\right|^{2} \prod_{n} d \theta_{n}$. Introducing Lagrange multipliers for the constraints, we obtain

$$
p\left(\theta_{1}, \theta_{2}, . . \theta_{N}\right) \prod_{n} d \theta_{n}=\prod_{r<s}\left|e^{i \theta_{s}}-e^{i \theta_{r}}\right|^{2} \prod_{n} w\left(\theta_{n}\right) d \theta_{n}
$$

where the positive weight factor is

$$
w(\theta)=\exp \left[\sum \lambda_{r} \cos r\left(\theta-\vartheta_{r}\right)\right] .
$$

The $\lambda$ 's and $\vartheta$ 's are the Lagrange multiplier parameters [two for each complex $\sigma_{r}$ ] needed to specify the traces and $\lambda_{r}$ vanishes for an unconstrained trace. We study $\bar{d}(\theta), R\left(\theta_{1}, \theta_{2}\right)$ obtained by integrating out all but one or two $\theta$ 's in Eq.(2).

The method of orthogonal polynomials is used [2], [15]. Polynomials $\phi_{n}(z)$ in $z=e^{i \theta}$ are found, satisfying $\frac{1}{2 \pi} \int d \theta w(\theta) \phi_{n}(z) \overline{\phi_{m}(z)}=\delta_{n m}$ where the bar indicates complex conjugation. This and subsequent integrals are over $[-\pi, \pi]$. Eq.(2) can be expressed $p\left(\theta_{1}, . . \theta_{N}\right)=\operatorname{det} M^{\dagger} M=\operatorname{det} K$ where $\sqrt{2 \pi} M_{n m}=$ $\Phi_{n}\left(\theta_{m}\right)=\sqrt{w\left(\theta_{m}\right)} \phi_{n}\left(\theta_{m}\right)$ and $K_{i j}=K\left(\theta_{i}, \theta_{j}\right)=$ $\frac{1}{2 \pi} \sum_{n=0}^{N-1} \Phi_{n}\left(\theta_{i}\right) \overline{\Phi_{n}\left(\theta_{j}\right)}$. The reason for writing $p$ in this form is that an integral over one variable of an $R \times R$ determinant of the form $\operatorname{det} K$ is proportional to the $(R-1) \times(R-1)$ determinant over the same argument. We thus find that

$$
\bar{d}(\theta)=K(\theta, \theta)
$$

and

$$
R\left(\theta_{1}, \theta_{2}\right)=\bar{d}\left(\theta_{1}\right) \bar{d}\left(\theta_{2}\right)-\left|K\left(\theta_{1}, \theta_{2}\right)\right|^{2}
$$

The sum in the definition of $K$ can be carried out [15]. The formula [Christoffel-Darboux] is 


$$
\sum_{n=0}^{N-1} \phi_{n}\left(z_{1}\right) \overline{\phi_{n}\left(z_{2}\right)}=\frac{z_{1}^{N} \bar{\phi}_{N}\left(z_{1}^{-1}\right) \bar{z}_{2}^{N} \phi_{N}\left(\bar{z}_{2}^{-1}\right)-\phi_{N}\left(z_{1}\right) \overline{\phi_{N}\left(z_{2}\right)}}{1-z_{1} \bar{z}_{2}} .
$$

$[\bar{\phi}(z) \equiv \overline{\phi(\bar{z})}$.$] We thus express everything in terms of the$ single polynomial $\phi_{N}$ [and $w$ ].

Given $w(\theta)$, satisfying certain conditions, a formula exists for $\phi_{N}$ for $N \rightarrow \infty$. The weight function of Eq.(3) is particularly congenial. If the exponent is a convergent series, the conditions are well satisfied.

The weight can be uniquely expressed by $w(\theta)=$ $|D(z)|^{2}$. Ambiguity in $D$ is removed by insisting that

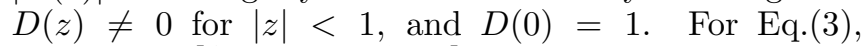
$D(z)=\exp \left[\frac{1}{2} \sum \lambda_{n} e^{-i n \vartheta_{n}} z^{n}\right]$. Asymptotically, for large $N$, the theorem [15] is that

$$
\phi_{N}(z) \approx z^{N}\left[\bar{D}\left(z^{-1}\right)\right]^{-1}
$$

Assuming asymptotia has been reached, we use Eq.(7) in Eq.(6) and Eq.(牙) to find, with L'Hôpital's rule, that

$$
\bar{d}(\theta) \approx \frac{N}{2 \pi}+\frac{1}{\pi} \operatorname{Re}\left[z \frac{D^{\prime}(z)}{D(z)}\right] .
$$

The integral over the nontrivial term vanishes. Thus

$$
\bar{d}(\theta)=\frac{N}{2 \pi}+\frac{1}{2 \pi} \sum n \lambda_{n} \cos n\left(\theta-\vartheta_{n}\right) .
$$

The trace conditions are $\sigma_{r}=\int d \theta e^{i r \theta} \bar{d}(\theta)=$ $\frac{1}{2} r \lambda_{r} e^{i r \vartheta_{r}}$, determining the Lagrange parameters. The mean density of states in energy is $\bar{d}(E)=\theta^{\prime} \bar{d}(\theta=0)=$ $\bar{d}+\frac{\theta^{\prime}}{\pi} \sum\left|\sigma_{r}\right| \cos \left(r \vartheta_{r}\right)$. Gutzwiller's density of states, expressed in terms of the traces of $T$ is

$$
\begin{aligned}
d_{G u t z}(E) & =\bar{d}+\frac{1}{\pi} \operatorname{Im} \frac{d}{d E} \sum \frac{1}{r} \sigma_{r}(E) \\
& \approx \bar{d}+\frac{1}{\pi} \sum \vartheta_{r}^{\prime}\left|\sigma_{r}\right| \cos \left(r \vartheta_{r}\right)
\end{aligned}
$$

where for pedagogical purposes we assume that $e^{i r \vartheta_{r}}$ is rapidly varying. If the derivative $\vartheta_{r}^{\prime} \approx \theta^{\prime}$, the leading terms of $d_{\text {Gutz }}$ coincide with the RMT $\bar{d}(E)$.

We thus have the interpretation that, at the level of the mean density, the random $T$ operator scheme is equivalent to moving the long wavelength part of $d_{o s c}$ into the mean density of states. The formulas above give a way of including this knowledge into a prediction of the correlation functions.

We next evaluate the two point correlations, using Eq.(5). Define $\psi(\theta)\left[=\frac{1}{2} \sum \lambda_{n} \sin n\left(\theta-\vartheta_{n}\right)\right]$ by $e^{i \psi(\theta)}=$ $D(z) /|D(z)|$. [For Dyson, $\psi=0$.] Then

$$
\begin{aligned}
\psi\left(\theta_{1}\right)-\psi\left(\theta_{2}\right) & =\sum \lambda_{n} \sin \frac{n}{2}\left(\theta_{1}-\theta_{2}\right) \cos \frac{n}{2}\left(\theta_{1}+\theta_{2}-2 \vartheta_{n}\right) \\
\left|K\left(\theta_{1}, \theta_{2}\right)\right|^{2} & =\left[\frac{\left.\sin \left[\frac{N}{2}\left(\theta_{1}-\theta_{2}\right)+\psi\left(\theta_{1}\right)-\psi\left(\theta_{2}\right)\right)\right]}{2 \pi \sin \frac{1}{2}\left(\theta_{1}-\theta_{2}\right)}\right]^{2}
\end{aligned}
$$

agreeing with Dyson if $\psi=0$. We wish to calculate

$$
\begin{aligned}
C(x) & =\left\langle C_{\epsilon}(x)\right\rangle_{W}=\left\langle\left\langle\frac{1}{d^{2}} d\left(\epsilon+\frac{x}{2 d}\right) d\left(\epsilon-\frac{x}{2 d}\right)\right\rangle\right\rangle_{W} \\
& =\left\langle\left\langle\frac{1}{d^{2}} \sum_{a, b} \delta\left(\epsilon+\frac{x}{2 d}-E_{a}\right) \delta\left(\epsilon-\frac{x}{2 d}-E_{b}\right)\right\rangle\right\rangle_{W} \\
& =\left\langle R\left(\epsilon+\frac{x}{2 d}, \epsilon-\frac{x}{2 d}\right)+\frac{1}{d} \delta\left(\frac{x}{d}\right)\right\rangle_{W}
\end{aligned}
$$

The outer average is over the energy range $E \pm W$. The inner average is over the random matrix ensemble.

We assume as above that $\theta_{a}(E)=\left(E-E_{a}\right) \theta^{\prime}$. Then

$$
R\left(E_{1}, E_{2}\right)=\left(\frac{\theta^{\prime}}{d}\right)^{2} R\left(\left(E-E_{1}\right) \theta^{\prime},\left(E-E_{2}\right) \theta^{\prime}\right)
$$

Using this in Eq.(12), with $\epsilon=E$ we obtain

$$
\begin{aligned}
C_{\epsilon}(x)= & \frac{1}{\bar{d}} \delta\left(\frac{x}{\bar{d}}\right)+\left(\frac{\theta^{\prime}}{\bar{d}}\right)^{2} \bar{d}\left(-\frac{\theta^{\prime} x}{2 \bar{d}}\right) \bar{d}\left(\frac{\theta^{\prime} x}{2 \bar{d}}\right)- \\
& {\left[\frac{\sin \left[\frac{\theta^{\prime} N x}{2 d}+\sum \lambda_{n} \sin \frac{n \theta^{\prime} x}{2 d} \cos n \vartheta_{n}\right]}{\frac{2 \pi \bar{d}}{\theta^{\prime}} \sin \frac{\theta^{\prime} x}{2 d}}\right]^{2} }
\end{aligned}
$$

Since $\theta^{\prime} / \bar{d}=2 \pi / N$ is small, we find (suppressing $\delta(x)$ )

$$
\begin{aligned}
C_{\epsilon}(x)=(1 & \left.+\sum c_{n+}\right)\left(1+\sum c_{m-}\right) \\
& -\left[\frac{\sin \pi x\left(1+\sum \frac{n \lambda_{n}}{N} \cos n \vartheta_{n}\right)}{\pi x}\right]^{2}
\end{aligned}
$$

where $c_{n \pm}(x)=\frac{n \lambda_{n}}{N} \cos n\left(\frac{\theta^{\prime} x}{2 d} \pm \vartheta_{n}\right)$

We now perform the energy average. Assume that only the $\vartheta_{n}$ 's vary significantly, and that they can be averaged independently. This gives the DA for the squared terms $c_{n+} c_{n-}$ in the first line of Eq.15), equivalent to that found by Berry [16]. We call this term $C_{B}(x)=\frac{1}{2} \sum\left(\frac{n \lambda_{n}}{N}\right)^{2} \cos \frac{2 \pi n}{N} x$. Let $\rho(y)=\rho(-y)=$ $\left\langle\delta\left(y-\sum \frac{n \lambda_{n}}{N} \cos n \vartheta_{n}\right)\right\rangle_{W}$ be the distribution of the correction term in the second line of Eq.(15). Its width is of order $N^{-1}$. The form factor [16] is

$$
\begin{aligned}
k(t) \equiv & 1+\int d x e^{2 \pi i t x} C(x)=\sum\left(\frac{n \lambda_{n}}{2 N}\right)^{2} \delta\left(|t|-t_{n}\right) \\
& +\left(1-\int_{|t|-1}^{\infty} d y \int_{y}^{\infty} d y^{\prime} \rho\left(y^{\prime}\right)\right)
\end{aligned}
$$

where $t_{n}(=n / N)$ is the period of the orbits associated with the $n$ 'th trace. The first term is a simulation of Berry's DA result for the short orbits. The second term rounds off the sharp corner found in standard RMT, [where $\rho=\delta(y)$.] The magnitude and width of the roundoff are both $\sim N^{-1}$. Since $\rho \geq 0$, we do not find the suggested [12] oscillations in $k(t)$ near $t=1$.

This expression mostly agrees with what is believed to be the correct form of $k(t)$. For large $|t|$, the discreteness of the spectrum dominates. For $|t| \sim t_{c} \sim N^{-1}$, the DA 
applied to Gutzwiller's expression gives spikes when $|t|$ is at a period of a short periodic orbit. We should improve our elementary estimate to replace $C_{B}$ as given above by Berry's result, and we should also make the corresponding change in $\psi(\theta)$. For $t_{c}<t<<1$, the DA applies and the sum rule [9] gives a straight line of unit slope. There is then structure near $|t|=1$ which is a consequence of the short period structure. The area removed under $k(t)$ near $t=1$ just compensates the area gained from the spikes at $t \sim t_{c}$, a consequence of $R(\theta, \theta)=0$. It's unclear whether resurgence plays a role in producing structure near $|t|=1$, as has been suggested [12. Our formulation contains all the ingredients needed for resurgence. RMT fluctuations in $\operatorname{det} T$ may wash out this effect. It seems rather that the energy average produces its biggest effect at $|t|=1$ because of the kink there.

The replacement $\sin \pi n x / N \rightarrow \pi n x / N$ in obtaining Eq.(15) from Eq.114) is not necessary. Averaging as before, we find

$$
\begin{gathered}
\left\langle\cos \left(2 \pi x+2 \sum \lambda_{n} \sin \frac{\pi n x}{N} \cos n \vartheta_{n}\right)\right\rangle_{W} \\
\approx \cos 2 \pi x \prod\left(1-\lambda_{n}^{2} \sin ^{2} \frac{\pi n x}{N}\right) \equiv x^{2} \mathcal{D}(x) \cos 2 \pi x
\end{gathered}
$$

and we have assumed $\lambda_{n}$ small. Then

$$
C(x)=\frac{1}{2 \pi^{2}}\left(-\frac{1}{2} \frac{\partial^{2} \ln \mathcal{D}(x)}{\partial x^{2}}+\mathcal{D}(x) \cos 2 \pi x\right),
$$

the relation found by SS [12]. With Eq.(17) small structures near $|t|=1$ decorate the result of Eq.(16) but the result is still monotonic.

It is not clear under what conditions Eq. (18) holds. The SS result is derived for large $x$, while ours is for small $\lambda_{n}$, in other words, for rather unstable orbits. The $\mathcal{D}(x)$ corresponding to a distribution $\rho$, is $\mathcal{D}(x)=$ $x^{-2} \int d y \rho(y) \cos 2 \pi x y$, but the term with $\ln \mathcal{D}$ does not reproduce $C_{B}$. The $\mathcal{D}$ of $[12]$ is derived from $C_{B}$ as a correction to the sum rule: it corresponds to a nonpositive 'distribution' $\rho(y) \propto \cos 2 \pi \gamma_{2} y \exp \left(-2 \pi \gamma_{1}|y|\right)$ where $\gamma_{1}+i \gamma_{2}$ is the lowest nonvanishing eigenvalue of the Perron-Frobenius operator. It of course does not give the spiky structure of $k(t)$ at $t \sim t_{c}$.

To summarize, an application of random matrix theory to Bogomolny's $T$ operator, one which retains knowledge of the short periodic orbits, leads to a relatively simple theory which gives reasonable results for the density of states and for the two point correlations. It does not agree in every detail with previously obtained results, however. This may be due to approximations we have made as well as the fact we have not specifically considered orbits of length of order $\gamma_{1}^{-1}>t_{c}$.

In this note, we have not worked out what happens for finite $N$, although the results should be meaningful. One can find the results for higher correlations directly. We don't expect them, or the corrections to the nearest neighbor level spacing, to be very illuminating, however. We hope to extend the results to other symmetry classes, and to parametric correlations. It should also be possible to compare statistics of eigenphases directly with this theory, in the case of quantum maps, [and indeed the $T$ operator is the quantum Poincaré map.]

We thank O. Bohigas and P. Lebœuf for extensive discussion and for hospitality at the INP, and E. Bogomolny for informing us of his unpublished work. We thank J. Bellissard for hospitality at the Université Paul Sabatier.

a Permanent address: Department of Physics, University of Maryland, College Park, MD 20742

$b$ Unité de Recherche Universités Paris 11 et Paris 6, Associée au CNRS.

c $\quad$ Unité Mixte de Recherche 5626

[1] M. C. Gutzwiller, Chaos in Classical and Quantum Mechanics, (Springer-Verlag, New York, 1991); Chaos and Quantum Physics, Proceedings of the Les Houches Summer School, Session LII, M. J. Giannoni, A. Voros and J. Zinn-Justin, eds. (Elsevier, New York, 1991); B. Eckhardt, Physics Reports 163,205 (1988).

[2] M. L. Mehta, Random Matrices, Academic Press (New York, 1990).

[3] M. C. Gutzwiller, J. Math. Phys. 12,343 (1971); R. Balian and C. Bloch, Ann. Physics (New York) 85,514 (1979).

[4] A. Voros, J. Phys. A21, 685-692(1988).

[5] M. V. Berry and J. P. Keating, J. Phys. 23, 4839 (1990); M. V. Berry and J. P. Keating, Proc. Roy. Soc.(London) A437, 151-173 (1992).

[6] E. B. Bogomolny, Nonlinearity 5, 805 (1992).

[7] E. Doron and U. Smilansky, Phys. Rev. Letters 68,1255 (1992); B. Dietz and U. Smilansky, Chaos 3(4),581 (1993).

[8] B. Georgeot and R. E. Prange, Phys. Rev. Lett. 74, 2851 (1995).

[9] J.H. Hannay and A.M. Ozorio de Almeida, J. Math. Phys. A17, 3429 (1984).

[10] N. Argaman et. al., Phys. Rev. Lett. 71, 4326 (1993).

[11] E. B. Bogomolny and J.P. Keating, (preprint, 1996).

[12] O. Agam, B. L. Altshuler, and A. V. Andreev, Phys. Rev. Lett. 75, 4389 (1995), and O. Agam et.al. preprint, 1996. Also see O. Agam and S. Fishman, Phys. Rev. Lett. 76, 726 (1996), A. L. Andreev and B. L. Altshuler, Phys. Rev. Lett. 75, 902 (1995).

[13] R. Balian, Nuovo Cimento 57, 290 (1968).

[14] K. A. Muttalib and M.E.H. Ismail, J. Phys. A: Math. Gen. 28, L541-L548 (1996); H. U. Baranger and P. A. Mello, Phys. Rev. Lett. 73, 142 (1994).

[15] G. Szegö, Orthogonal Polynomials, Amer. Math. Soc. Colloq. Pubs. (Providence, R.I. 1975).

[16] M. V. Berry, Proc. R. Soc. Lond. A400, 229 (1985). 